\title{
Decay constants of heavy mesons from QCD sum rules
}

\section{Wolfgang Lucha}

HEPHY, Austrian Academy of Sciences, Nikolsdorfergasse 18, A-1050 Vienna, Austria

E-mail: wolfgang.lucha@oeaw.ac.at

\section{Dmitri Melikhov*}

HEPHY, Austrian Academy of Sciences, Nikolsdorfergasse 18, A-1050 Vienna, Austria

Faculty of Physics, University of Vienna, Boltzmanngasse 5, A-1090 Vienna, Austria

SINP, Moscow State University, 119991, Moscow, Russia

E-mail: dmitri_melikhov@gmx.de

\section{Silvano Simula}

INFN, Sezione di Roma III, Via della Vasca Navale 84, I-00146, Roma, Italy

E-mail: Simula@roma3.infn.it

We present a sum-rule extraction of the decay constants of the $D, D_{s}, B$, and $B_{s}$ mesons from the two-point correlator of heavy-light pseudoscalar currents [1]]. Our main emphasis is laid on the control over the uncertainties in the decay constants, related both to the input QCD parameters and to the limited accuracy of the method of sum rules. Gaining this control has become possible due to the application of our novel procedure for extracting hadron observables based on a dual threshold depending on the Borel parameter. For charmed mesons, we obtain $f_{D}=(206.2 \pm$ $\left.7.3_{(\mathrm{OPE})} \pm 5.1_{(\mathrm{syst})}\right) \mathrm{MeV}$ and $f_{D_{s}}=\left(245.3 \pm 15.7_{(\mathrm{OPE})} \pm 4.5_{(\text {syst })}\right) \mathrm{MeV}$. For beauty mesons, the decay constants turn out to be extremely sensitive to the precise value of the $\overline{\mathrm{MS}}$ mass of the $b$ quark, $\bar{m}_{b}\left(\bar{m}_{b}\right)$. By requiring our sum-rule estimate to match the average of lattice determinations of $f_{B}$, a very accurate value is extracted, $\bar{m}_{b}\left(\bar{m}_{b}\right)=(4.245 \pm 0.025) \mathrm{GeV}$, yielding $f_{B}=(193.4 \pm$ $\left.12.3_{(\mathrm{OPE})} \pm 4.3_{(\text {syst })}\right) \mathrm{MeV}$ and $f_{B_{s}}=\left(232.5 \pm 18.6_{(\mathrm{OPE})} \pm 2.4_{(\text {syst })}\right) \mathrm{MeV}$.

35th International Conference of High Energy Physics - ICHEP2010,

July 22-28, 2010

Paris France

\footnotetext{
${ }^{*}$ Speaker.
} 


\section{Introduction}

The extraction of the decay constants of ground-state heavy pseudoscalar mesons within the method of QCD sum rules [2] is a complicated problem: First, one should derive a reliable operator product expansion (OPE) for the Borelized correlation function of two pseudoscalar heavy-light currents. We make use of the OPE for this correlator to three-loop accuracy [B], reshuffled in terms of the $\overline{\mathrm{MS}}$ heavy-quark mass, in which case the perturbative expansion exhibits a reasonable convergence [4]. Second, the knowledge of the truncated OPE for the correlator - even if the parameters of this OPE are known precisely - allows extracting the characteristics of the bound state with a limited accuracy which reflects the intrinsic uncertainty of the method of QCD sum rules. The control over this uncertainty is a very subtle problem [5].

Recently, we have formulated a new approach to extract the ground-state parameters from the correlator which enables such a control [6]. Let us briefly recall the essential features of our approach: The quark-hadron duality assumption leads to a relation between ground-state contribution and OPE for the correlator with a cut at some effective continuum threshold $s_{\text {eff: }}$ :

$$
f_{Q}^{2} M_{Q}^{4} e^{-M_{Q}^{2} \tau}=\Pi_{\text {dual }}\left(\tau, s_{\text {eff }}(\tau)\right) \equiv \int_{\left(m_{Q}+m\right)^{2}}^{s_{\text {eff }}(\tau)} d s e^{-s \tau} \rho_{\text {pert }}(s)+\Pi_{\text {power }}(\tau) .
$$

Evidently, in order to extract the decay constant one has to fix the effective continuum threshold $s_{\text {eff }}$. Moreover, as is obvious from (1.1) $s_{\text {eff }}$ must be a function of $\tau$, otherwise the 1.h.s. and the r.h.s. of (1.1) exhibit a different $\tau$-behaviour. The exact effective continuum threshold, corresponding to exact values of hadron mass and decay constant on the 1.h.s., is of course not known. Therefore, the extraction of hadron parameters from the sum rule consists in attempting (i) to find a reasonable approximation to the exact threshold and (ii) to control the accuracy of this approximation.

Let us introduce the dual invariant mass $M_{\text {dual }}$ and the dual decay constant $f_{\text {dual }}$ by the relations

$$
\begin{aligned}
M_{\text {dual }}^{2}(\tau) & \equiv-\frac{d}{d \tau} \log \Pi_{\text {dual }}\left(\tau, s_{\text {eff }}(\tau)\right), \\
f_{\text {dual }}^{2}(\tau) & \equiv M_{Q}^{-4} e^{M_{Q}^{2} \tau} \Pi_{\text {dual }}\left(\tau, s_{\text {eff }}(\tau)\right) .
\end{aligned}
$$

If the mass of the ground state is known, any deviation of the dual mass from the actual mass of the ground state yields an indication of the excited-state contributions picked up by the dual correlator. Assuming a particular functional form of the effective threshold and requiring the least deviation of the dual mass (1.2) from the actual mass in the $\tau$-window leads to a variational solution for the effective threshold; as soon as the latter has been fixed we derive the decay constant from (1.3). The standard assumption for the effective threshold is a $\tau$-independent constant. In addition to this approximation, we have considered polynomials in $\tau$. Reproducing the actual mass considerably improves for $\tau$-dependent thresholds. This means that a dual correlator with $\tau$-dependent threshold isolates the ground-state contribution much better and is less contaminated by the excited states than a dual correlator with standard $\tau$-independent threshold. As consequence, the accuracy of extracted hadron observables improves considerably. Recent experience from potential models reveals that the band of values obtained from the linear, quadratic, and cubic Ansätze for the effective threshold encompasses the true value of the decay constant [6]. Moreover, we could show that the extraction procedures in quantum mechanics and in QCD are even quantitatively rather similar [7]. 
This contribution reports our recent results [1] for the heavy-meson decay constants.

\section{Decay constants of the $D$ and $D_{s}$ mesons}

The application of our extraction procedures leads to the following results for charmed mesons:

$$
\begin{aligned}
f_{D} & =\left(206.2 \pm 7.3_{(\mathrm{OPE})} \pm 5.1_{(\text {(syst })}\right) \mathrm{MeV} \\
f_{D_{s}} & =\left(245.3 \pm 15.7_{(\mathrm{OPE})} \pm 4.5_{(\text {(syst })}\right) \mathrm{MeV} .
\end{aligned}
$$

The OPE-related error is obtained by the bootstrap allowing for the variation of all QCD parameters (i.e., quark masses, $\alpha_{s}$, condensates) in the relevant ranges. One observes a perfect agreement of our predictions with the respective lattice results (Fig. 1). It should be emphasized that our $\tau$-dependent threshold is a crucial ingredient for a successful extraction of the decay constants from the sum rule. Obviously, the standard $\tau$-independent approximation yields a much lower value for $f_{D}$ lying rather far from the experimental data and from the lattice results.
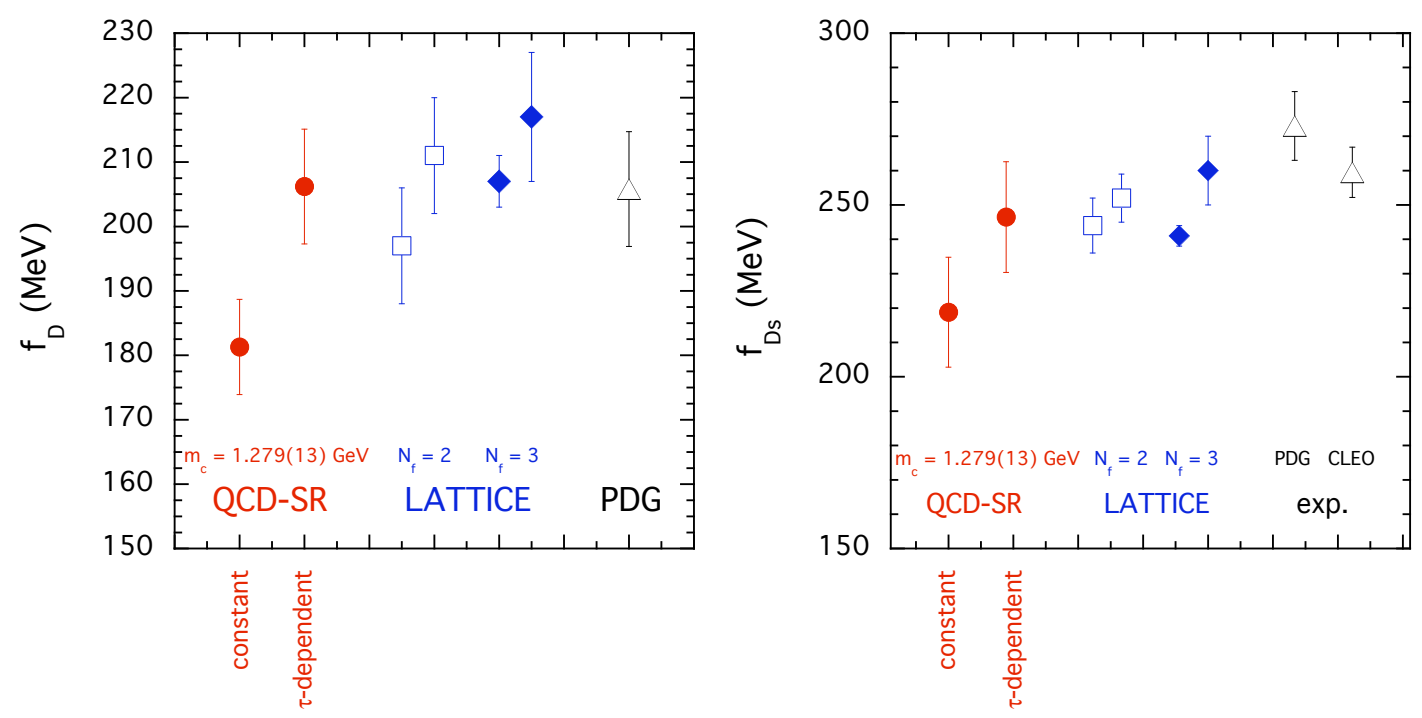

Figure 1: Comparison of our results for $f_{D}$ and $f_{D_{s}}$ with lattice results; for a detailed list of references, cf. [1].

\section{Decay constants of the $B$ and $B_{s}$ mesons}

The values of the beauty-meson decay constants extracted from QCD sum rules are extremely sensitive to the precise value of $\bar{m}_{b}\left(\bar{m}_{b}\right)$. For instance, the range $\bar{m}_{b}\left(\bar{m}_{b}\right)=(4.163 \pm 0.016) \mathrm{GeV}$ [8] yields results for the decay constants that are barely compatible with the lattice calculations (Fig. 2). Requiring our sum-rule result for $f_{B}$ to match the average of the lattice determinations entails a rather precise value of the $b$-quark mass

$$
\bar{m}_{b}\left(\bar{m}_{b}\right)=(4.245 \pm 0.025) \mathrm{GeV} \text {. }
$$

Our sum-rule estimates for $f_{B}$ and $f_{B_{s}}$ corresponding to this value of the $b$-quark mass are

$$
\begin{aligned}
f_{B} & =\left(193.4 \pm 12.3_{(\mathrm{OPE})} \pm 4.3_{(\text {syst })}\right) \mathrm{MeV}, \\
f_{B_{s}} & =\left(232.5 \pm 18.6_{(\mathrm{OPE})} \pm 2.4_{(\text {syst }}\right) \mathrm{MeV} .
\end{aligned}
$$



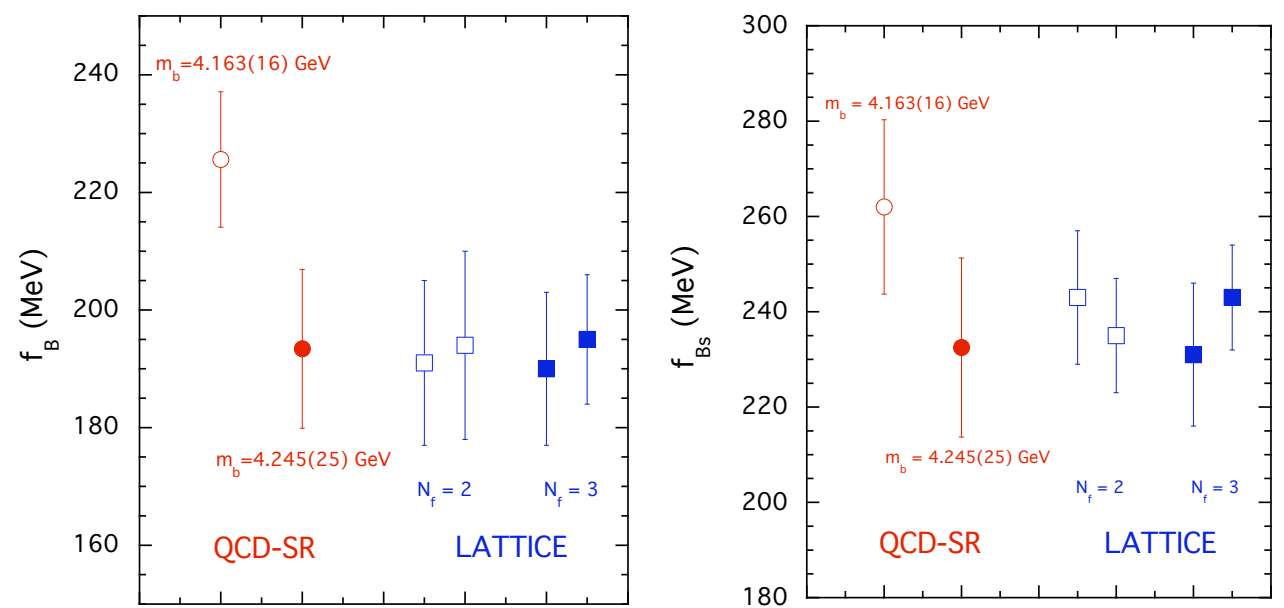

Figure 2: Comparison of our results for $f_{B}$ and $f_{B_{s}}$ with lattice results; for a detailed list of references, cf. [1].

\section{Conclusions}

1. Our study of charmed mesons clearly demonstrates that the use of Borel-parameter-dependent thresholds leads to two essential improvements: (i) The accuracy of decay constants extracted from sum rules is considerably improved. (ii) It has become possible to obtain realistic systematic errors and to reduce their values to the level of a few percent. The application of our prescription brings the QCD sum-rule results into perfect agreement with the findings of both lattice QCD and experiment.

2. The beauty-meson decay constants are extremely sensitive to the precise value of $\bar{m}_{b}\left(\bar{m}_{b}\right)$; matching the results from QCD sum rules for $f_{B}$ to the average of the lattice evaluations allows us to provide a rather accurate estimate of the $b$-quark mass. Our $m_{b}$ value is in good agreement with several lattice results but, interestingly, does not overlap with the recent accurate determination [8] (for details, consult Ref. [1]).

Acknowledgments. D.M. was supported by the Austrian Science Fund (FWF), project no. P20573.

\section{References}

[1] W. Lucha, D. Melikhov, and S. Simula, arXiv:1008.2698 [hep-ph].

[2] M. Shifman, A. Vainshtein, and V. Zakharov, Nucl. Phys. B 147, 385 (1979).

[3] K. G. Chetyrkin and M. Steinhauser, Phys. Lett. B 502, 104 (2001); Eur. Phys. J. C 21, 319 (2001).

[4] M. Jamin and B. O. Lange, Phys. Rev. D 65, 056005 (2002).

[5] W. Lucha, D. Melikhov, and S. Simula, Phys. Rev. D 76, 036002 (2007); Phys. Lett. B 657, 148 (2007); Phys. Atom. Nucl. 71, 1461 (2008).

[6] W. Lucha, D. Melikhov, and S. Simula, Phys. Rev. D 79, 096011 (2009); J. Phys. G 37, 035003 (2010); W. Lucha, D. Melikhov, H. Sazdjian, and S. Simula, Phys. Rev. D 80, 114028 (2009).

[7] W. Lucha, D. Melikhov, and S. Simula, Phys. Lett. B 687, 48 (2010); arXiv:1003.1463 [hep-ph].

[8] K. G. Chetyrkin et al., Phys. Rev. D 80, 074010 (2009). 